\title{
Suplementação de prebióticos e probióticos em crianças autistas: revisão integrativa
}

\author{
Supplementation of prebiotics and probiotics in autistic children: integrative review \\ Suplementación de prebióticos y probióticos en niños autistas: una revisión integradora
}

Recebido: 29/11/2021 | Revisado: 06/12/2021 | Aceito: 21/12/2021 | Publicado: 03/01/2022

Thaynara Lays Sales Brandão

ORCID: https://orcid.org/0000-0002-5088-5746 Faculdade de Comunicação, Tecnologia e Turismo de Olinda, Brasil
E-mail: thaynaralays@gmail.com

Julia Carolina Lopes Silva

ORCID: https://orcid.org/0000-0002-9682-6581

Universidade Federal de Pernambuco, Brasil E-mail: julia.lopes@ufpe.br

Sarah Évelin Dias Campos

ORCID: https://orcid.org/0000-0003-2461-400X Faculdade de Comunicação, Tecnologia e Turismo de Olinda, Brasil E-mail: diassaarah@gmail.com

Jakeline Olindina Francelino

ORCID: https://orcid.org/0000-0002-9851-5269

Faculdade de Comunicação, Tecnologia e Turismo de Olinda, Brasil

E-mail: jakelineolindina.prof@hotmail.com

\begin{abstract}
Resumo
Objetivo: Avaliar o uso da suplementação de probióticos e prebióticos em crianças autistas. Metodologia: Foram identificados artigos nas bases de dados PubMed e SciELO. Para a localização dos estudos foram utilizados descritores em inglês cadastrados no Descritores em Ciências da Saúde (DeCS): Prebiotics. Probiotics. Autism Spectrum Disorder. Gut microbiota. Bifidobacterium. A busca limitou-se a artigos em inglês e compreendeu os anos de publicação entre 2015-2021. Resultados: Um total de 6 ensaios clínicos realizados em crianças com autismo foram revisados sistematicamente. Com relação aos tipos de suplementações utilizadas foram 3 estudos que utilizaram probióticos, 2 que utilizaram prebióticos e probióticos e 1 que fez apenas o uso de prebióticos. Apesar da quantidade escassa de estudos, os mesmos tiveram resultados notáveis com relação à suplementação de probióticos e prebióticos em crianças com Transtorno do Espectro Autista. Em alguns estudos foi possível observar a melhora na gravidade do autismo e melhorias com relação ao comportamento antissocial, já em outros houve o aumento de bactérias benéficas e diminuição das patogênicas no trato gastrintestinal levando a melhora de sintomas gastrointestinais recorrentes. Conclusão: O uso de prebióticos e probióticos apresentam resultados significativos em crianças com TEA, sendo uma terapia alternativa, promissora e complementar. Ainda assim, há a necessidade que mais ensaios clínicos sejam realizados acerca do assunto, pois são escassos e com amostras pequenas.
\end{abstract}

Palavras-chave: Autismo; Probióticos; Prebióticos; Criança.

\begin{abstract}
Objective: To evaluate the use of probiotics and prebiotics supplementation in autistic children. Methodology: Articles were identified in the PubMed and SciELO databases. In order to locate the studies, descriptors in English obtained from the Health Sciences Descriptors (DeCS), were used: Prebiotics. Probiotics. Autism Spectrum Disorder. Gut microbiota. Bifidobacterium. The search was limited to articles in Portuguese and English and comprised the years of publication between 2015-2021. Results: A total of 6 clinical trials conducted on children with autism have been systematically reviewed. Regarding the types of supplements used, there were 3 studies that used probiotics, 2 that used prebiotics and probiotics and 1 that made only the use of prebiotics. Despite the scarce amount of studies, they have had remarkable results in relation to the supplementation of probiotics and prebiotics in children with ASD. In some studies, it was possible to observe an improvement in the severity of autism and improvements in relation to antisocial behavior, while in others, there was an increase in beneficial bacteria and a decrease in pathogens in the gastrointestinal tract, leading to an improvement in recurrent gastrointestinal symptoms. Conclusion: The use of prebiotics and probiotics has shown significant results in children with ASD, being an alternative, promising and complementary therapy. Still, there is a need for more clinical trials to be carried out on the subject, as they are scarce and with small samples.
\end{abstract}

Keywords: Autism; Probiotics; Prebiotics; Child. 


\begin{abstract}
Resumen
Objetivo: Evaluar el uso de suplementos probióticos y prebióticos en niños autistas. Metodología: Se identificaron artículos en las bases de datos PubMed y SciELO. Para la ubicación de los estudios se utilizaron descriptores en inglés registrados en los Descriptores en Ciencias de la Salud (DeCS): Prebióticos. Probióticos. Desorden del espectro autista. Microbiota intestinal. Bifidobacterium. La búsqueda se limitó a artículos en inglés y cubrió los años de publicación entre 2015-2021. Resultados: Se revisaron sistemáticamente un total de 6 ensayos clínicos realizados en niños con autismo. En cuanto a los tipos de suplementación utilizados, hubo 3 estudios que utilizaron probióticos, 2 que utilizaron prebióticos y probióticos, y 1 que solo utilizó prebióticos. A pesar de la escasa cantidad de estudios, han tenido resultados notables con respecto a la suplementación de probióticos y prebióticos en niños con trastorno del espectro autista. En algunos estudios se pudo observar una mejora en la severidad del autismo y mejoras en relación a la conducta antisocial, mientras que en otros hubo un aumento de bacterias benéficas y una disminución de patógenos en el tracto gastrointestinal, conduciendo a una mejoría en las recurrencias gastrointestinales. síntomas. Conclusión: El uso de prebióticos y probióticos ha mostrado resultados significativos en niños con TEA, siendo una terapia alternativa, prometedora y complementaria. Aún así, es necesario realizar más ensayos clínicos sobre el tema, ya que son escasos y con pequeñas muestras.
\end{abstract}

Palabras clave: Autismo; Probióticos; Prebióticos; Niño.

\title{
1. Introdução
}

O Transtorno do Espectro Autista (TEA) é uma síndrome que é caracterizada por alterações atípicas no sistema nervoso relacionado ao desenvolvimento, sendo designado como desordem do desenvolvimento difuso. Pacientes com TEA apresentam sintomas como: déficit de interação social, comprometimento verbal, comportamentos repetitivos e restritivos possuindo interesses limitados, podendo também apresentar, comportamento agressivo (consigo e terceiros) e estímulos sensoriais aguçados (Hirota et al., 2021).

Alterações gastrointestinais como constipação, diarreia, doença celíaca, intolerância alimentar e disbiose intestinal ocorrem comumente neste público, interferindo na capacidade de ingestão e absorção tanto do alimento como seu nutriente, para melhor compreensão do porquê esses sintomas serem presentes, um estudo investigou a composição da microbiota intestinal de crianças com TEA em comparação a neurotípicos e afirmam que indivíduos autistas possuem concentrações maiores de bactérias patogênicas como Clostridium, menor número de Bacteroides / Firmicutes, e aumento de Lactobacillus e Desulfovibrio (Strati et al., 2017; Almeida et al., 2018).

O desequilíbrio da comunicação bidirecional entre o intestino e o cérebro pode ter relação direta com os agravos do TEA, visto que a microbiota intestinal pode ter um papel na modulação imunológica e funções gastrointestinais (Santochi et al., 2020). A composição alterada da microbiota intestinal pode reduzir a integridade da barreira intestinal havendo a absorção de toxinas, tendo assim o aumento de biomarcadores inflamatórios (Silva et al., 2020).

O uso de probióticos e prebióticos vem sendo analisado para melhora de sintomas gastrointestinais e comportamentais no autismo, visto que os probióticos têm a capacidade de modular a microbiota intestinal, e os prebióticos são substratos utilizados pelos microrganismos intestinais para o aumento de bactérias benéficas, podendo trazer respostas positivas para os infantis que possuem esta condição (Grimaldi et al., 2017; Marco et al., 2021). Com isto, o objetivo deste estudo foi realizar uma análise integrativa sobre a suplementação de probióticos e prebióticos em crianças autistas.

\section{Metodologia}

Este estudo trata-se de uma pesquisa de revisão integrativa, desenvolvida por meio da estratégia PICOS que representa um acrônimo para Paciente, Intervenção, Comparação (controle), Outcomes (desfecho) e Study design (desenho do estudo) (Liberati et al., 2009). Cada enfoque da PICOS equivaleu aos seguintes elementos: (P) crianças com transtorno do espectro autista e alterações na microbiota intestinal; (I) suplementação de prebióticos e probióticos; (C) placebo e crossover; (O) avaliação de estudos que correlacionaram a intervenção de prebióticos e probióticos na melhora do quadro de crianças com transtorno do espectro autista; (S) ensaios clínicos. Essa estratégia resultou na delimitação da seguinte pergunta norteadora: A 
suplementação de prebióticos e probióticos em crianças autistas pode ser eficaz na melhora da sua sintomatologia?

Nas buscas, conforme a figura 1, foram utilizados descritores em inglês cadastrados no Descritores em Ciências da Saúde (DeCS): ((“Prebiotics”. OR "Probiotics”)) AND “Autism Spectrum Disorder”. "Gut microbiota”. ("Lactobacillus” OR "Bifidobacterium") AND “Autism Spectrum Disorder". As bases de dados utilizadas foram: PubMed e SciELO. Os critérios de inclusão estabelecidos foram: ensaios clínicos publicados entre os anos de: 2015 - 2021, sendo todos realizados em crianças com diagnóstico clínico de TEA, tendo em sua temática os seguintes termos: autismo, prebióticos, probióticos, suplementação e microbiota. Com dados disponíveis sobre sintomas gastrointestinais/ comportamentais/ psiquiátricos. Sendo excluídos: Artigos de revisão, relatos de experiência, relatórios técnicos, resumos, trabalhos apresentados em eventos científicos, bem como artigos fora da temática deste estudo.

Para os critérios de elegibilidade, foi realizada uma leitura dos artigos na integra, sendo observados dados como: nome do autor, ano de publicação, delineamento da pesquisa, objetivos, perfil amostral intervenção e desfecho. Os estudos selecionados foram postos em uma tabela para facilitar a visualização dos resultados e relevância de cada um.

Figura 1: Fluxograma de elegibilidade dos artigos para revisão integrativa:

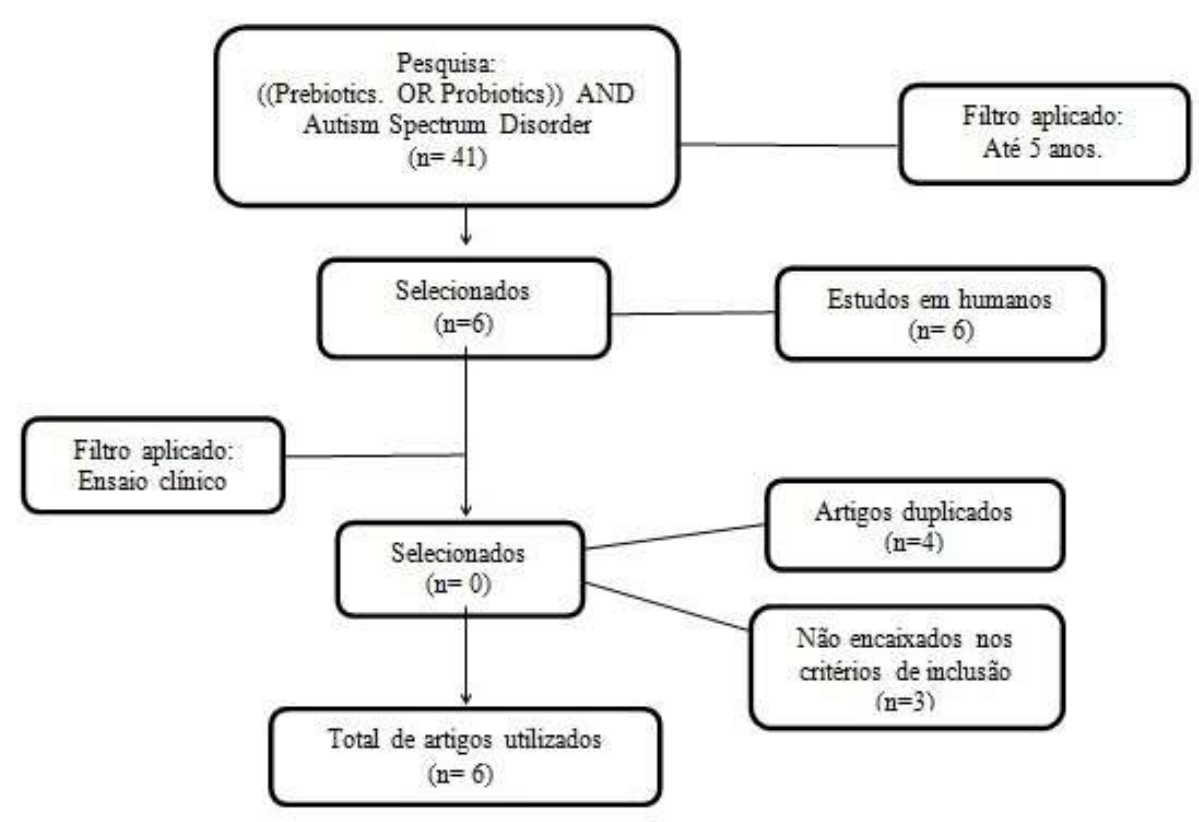

Fonte: Autores (2021).

\section{Resultados e Discussão}

Um total de 6 ensaios clínicos realizados em crianças com autismo foram revisados sistematicamente, conforme a Tabela 1. Com relação aos tipos de suplementações utilizadas foram 3 estudos que utilizaram probióticos, 2 que utilizaram prebióticos e probióticos e 1 que fez apenas o uso de prebióticos. 
Tabela 1: Síntese de artigos selecionados.

\section{Referência}

WANG, Ying et al., 2020.

LIU,Yen-Wenn et al, Público alvo: 2019.

Crianças com TEA

$(\mathrm{n}=71)$

$($ Placebo=35).

Idade: 7 - 15 anos.

SANCTUARY, M. Público alvo:

R. et al., 2019.

GRIMALDI, R. et al., 2018.

Crianças com TEA

e comorbidades

gastrointestinais

$(\mathrm{n}=8)$.

Idade: 2-11 anos.

Público alvo:

Crianças com TEA

$(n=30)$.

Idade: 4-11 anos.

SHAABAN, S. Y. et al., 2018.

\section{Suplementação}

\section{Resultados}

\author{
Prebiótico: Fruto-oligossacarídeo. \\ Probiótico: Bifidobacterium \\ infantisBi-26, Lactobacillus \\ rhamnosusHN001, Bifidobacterium \\ lactisBL-04 e Lactobacillus \\ paracaseiLPC-37); \\ Dosagem: $10^{10} \mathrm{UFC} / \mathrm{pacote} / \mathrm{dia}$;
}

Duração: 12 meses.

Probiótico: Lactobacillus plantarum

PS128;

Dosagem: $3 \times 10^{10}$ UFC / cápsula;

Duração: 4 semanas.

Prebiótico: PCB

Probiótico: B. Infantis;

Dosagem: 0,15 g/lb/ Kg/dia e 20

bilhões de UFC por dia;

Duração: 12 semanas.

Prebiótico: B-GOS®;

Dosagem: -

Duração: 6 semanas.

Probiótico: Lactobacillus acidophilus, Lactobacillus rhamnosus e Bifidobacteria longum; Dosagem: 5g/dia (100x10 $\left.10^{6} \mathrm{UFC} / \mathrm{g}\right)$;

Duração: 3 meses.
Grupo placebo não mostrou resultados, grupo PS128 mostrou melhora nominal em vários elementos, uso de objetos, ansiedade, hiperatividade / impulsividade. $\uparrow$ benéfico em indivíduos mais jovens ( 7 - 12 anos).

Ambas intervenções tiveram resultados positivos como, melhora comportamental; ganho de peso; $\downarrow$ sintomas GI; $\downarrow$ IL-13 e TNF$\alpha$.

$\mathrm{Na}$ intervenção foi constatado $\uparrow$ família Lachnospiraceae, $\downarrow$ dor abdominal e evacuação, melhorias no comportamento anti-social e mudanças nos metabólitos fecais e urinários.

$\uparrow$ nas contagens de colônias de bifidobactérias e lactobacilos, $\downarrow$ no peso corporal, bem como melhorias significativas na gravidade do autismo e sintomas gastrointestinais em início do estudo. comparação com a linha de base avaliada no 
TOMOVA, A. et al., Público alvo: 2015.

Público alvo:
Crianças com
autismo ( $\mathrm{n}=10)$
irmãos de autistas
$(\mathrm{n}=9)$ e neurotípicas
$(\mathrm{n}=10)$.

Idade: 2- 17 anos.
Probiótico: "Children Dophilus" (3 cepas de Lactobacillus (60\%), 2 cepas de Bifidumbactérias (25\%) e uma cepa de Streptococcus (15\%));

Dosagem: 3 cápsulas/ dia;

Duração: 4 meses. $\downarrow$ Firmicutes, $\uparrow$ razão Bacteroidetes/ Firmicutes para o nível de indivíduos saudáveis. $\downarrow$ Bifidobacterium atingindo o mesmo nível de neurotípicos. $\uparrow \quad 2 \mathrm{x} \quad$ Lactobacillus, $\downarrow$ Desulfovibrio (etiopatogenético) após a terapia.

Siglas e abreviações: $\uparrow$ (aumento); $\downarrow$ (diminuição); AGCC (Ácidos Graxos de Cadeia Curta); B-GOS® (Beta galacto-oligossacarídeo); GI (Gatrointestinal); PCB (Produto de Colostro Bovino); TEA (Transtorno do Espectro Autista); UFC (Unidade Formadora de Colônia). Fonte: Autores (2021).

Apesar da quantidade escassa de estudos, os mesmos tiveram resultados notáveis com relação à suplementação de probióticos e prebióticos em crianças com TEA. Em alguns estudos foi possível observar a melhora na gravidade do autismo e melhorias com relação ao comportamento antissocial, já em outros houve o aumento de bactérias benéficas e diminuição das patogênicas no trato gastrintestinal levando a melhora de sintomas gastrointestinais recorrentes (Tomova et al., 2015; Grimaldi et al., 2017; Shaaban et al., 2018; Liu et al., 2019; Sanctuary et al., 2019; Wang, 2020;).

No estudo feito por Liu e colaboradores (2019) apesar da intervenção ter tido um curto tempo de duração - quatro semanas - o uso do probiótico Lactobacillus plantarum PS128, ofereceu mais benefícios para as crianças autistas com idade entre 7 - 12 anos do que os com idade mais avançada, mostrando que o tratamento com probióticos pode ser eficaz quando a intervenção é feita de forma precoce.

Tomova e colaboradores (2015) notou que a gravidade do autismo e sintomas gastrointestinais podem estar ligados com o aumento dos níveis da citocina pró-inflamatória TNF- $\alpha$, pois após o uso de probióticos na análise fecal dos participantes teve-se a diminuição desta citocina e consequentemente houve a melhora nos escores de comportamento restritivos e repetitivos e melhora da população bacteriana na microbiota intestinal. Em outro estudo a suplementação de probióticos em conjunto com os prebióticos teve-se a redução das citocinas IL-13 e TNF- $\alpha$, observando também benefícios no comportamento social com relação à irritabilidade e esteriopatia e sintomas gastrointestinais, estes resultados podem estar relacionados com a redução da inflamação intestinal (Sanctuary et al., 2019).

No estudo realizado por Grimaldi e colaboradores (2017), a suplementação com Prebiótico B-GOS® (um galactooligossacarídeo) em 30 crianças com TEA de 4-11 anos de idade, fez com que houvessem melhorias nos sintomas antissociais e gastrointestinais, porém isso apenas foi notificado em participantes com dietas de exclusão de glúten e caseína. A mitigação de problemas comportamentais característicos do TEA também foi vista com a administração de um probiótico composto por 3 cepas - Lactobacillus acidophilus, Lactobacillus rhamnosus e Bifidobacteria longum- o perfil bacteriano apresentou aumentos nas colônias de bifidobactérias e lactobacilos, havendo também um menor número de Clostridium nas amostras fecais (Shaaban. et al., 2018).

A reversão do estado de disbiose pelo uso de probióticos e fruto-oligossacarídeos em indivíduos com autismo na análise feita por Wang e colaboradores (2020), foi apontada pelo aumento de Bifidobacteriales e B. Longun e supressão de Clostridium, antes da intervenção o grupo com TEA foi comparado com o grupo controle e foram percebidos diferentes níveis de Ácidos Gaxos de Cadeia Curta (AGCC) e neurotransmissores, os níveis de ácido acético, butírico e propiônico foram significativamente menores no grupo com TEA, após a intervenção teve-se um aumento de AGCC próximo aos dos níveis do grupo controle, redução da gravidade do autismo e diminuição de sintomas gastrintestinais. 


\section{Definição do autismo, etiologia e epidemiologia}

De acordo a World Health Organization, em média, a cada 160 crianças no mundo, 1 é diagnosticada com TEA, tendo como prevalência o sexo masculino (WHO, 2020). Os dados mais recentes sobre TEA no Brasil são os do censo demográfico do Instituto Brasileiro de Geografia e Estatística, onde a estimativa do número de crianças autistas é de 454.706, com uma taxa de prevalência de 1 para 150, na proporção de 3 homens para 1 mulher (IBGE, 2010).

O Transtorno do Espectro Autista é um distúrbio do neurodesenvolvimento caracterizado por sintomas como dificuldade persistente ao se comunicar de forma recíproca e ao interagir socialmente, possuindo interesses, comportamentos ou atividades com padrões restritos e repetitivos, sendo estes presentes na infância ocorrendo de acordo com a gravidade da condição autista, nível de desenvolvimento e idade cronológica, sendo esta a razão para o uso do termo espectro. O seu diagnóstico geralmente é dado aos três anos de idade, período que os pais preocupam-se com o atraso de linguagem e desenvolvimento social do infante, porém pode também haver o diagnóstico precoce caso os sintomas sejam mais evidentes, podendo ser observados a ecolalia, demonstração de movimentos estereotipados, como, balançar as mãos, andar com a ponta dos pés ou passar os dedos perto dos olhos (American Psychiatric Association, 2014; Parmeggiani; Corinaldesi; Posar, 2019).

A seletividade alimentar é geralmente muito presente na infância, principalmente no início, tanto em crianças neurotípicas (rótulo utilizado para pessoas que não tem autismo ou outros transtornos psíquicos) como nas com TEA, porém nos autistas este caso é mais severo, visto que possuem os níveis de recusa alimentar bastante elevado, menor repertório alimentar e diversidade escassa na ingestão de alimentos como frutas e legumes (O'connor et al., 2005; Chistol et al., 2018).

O TEA engloba transtornos como o transtorno autista (autismo), a síndrome de Asperger (desenvolvimento da fala no tempo esperado, sem a presença de retardo mental), o transtorno desintegrativo da infância (desenvolvimento inicial normal relacionado à cognição, linguagem e comportamento, havendo a regressão entre 2 a 10 anos de idade, estabilizando-se sem haver regressão ou progresso) e o transtorno global do desenvolvimento sem outra especificação (quando as características não se aplicam em relação aos outros transtornos citados). Este transtorno possui níveis de severidade onde, são subdivididos em nível 1 (leve), cujo o indivíduo apenas requer suporte, nível 2 (moderado) que exige suporte substancial e nível 3 (severo) quando necessita de muito suporte substancial (American Psychiatric Association, 2014; Mendes et al., 2016).

Em relação a sua etiologia, o autismo possui causas multifatoriais que podem ser consideradas para seu desenvolvimento, as mais prevalentes são em relação a fatores ambientais e genéticos. Os ambientais levam em consideração a idade avançada dos pais na concepção, uso de certos medicamentos no período pré-natal (por exemplo: ácido valpróico), nascimento prematuro e baixo peso ao nascer (American Psychiatric Association, 2014; Sociedade Brasileira de Pediatria, 2019). No caso dos fatores genéticos, inúmeros estudos detectaram diversos genes que podem estar relacionados ao TEA, as principais anormalidades são encontradas em genes codificadores de proteínas sinápticas, sendo estas responsáveis por regular a transcrição de DNA ou tradução de mRNA, proteínas do citoesqueleto e da membrana, conexões intercelulares e maturação celular (Strasser et al., 2018).

\section{Fisiologia gastrointestinal e autismo}

Em um estudo feito por Silva e colaboradores (2020), cerca de 85,5\% da amostra de 39 crianças com TEA relataram a presença de alterações gastrointestinais, principalmente em indivíduos que apresentavam em sua dieta o consumo de glúten, caseína e ultraprocessados, também se notou que a constipação foi a alteração que teve maior incidência.

Alguns estudos que compararam a microbiota intestinal de crianças com TEA com pessoas neurotípicas observaram que é notória a menor diversidade de bactérias no trato gastrointestinal e até mesmo a presença de bactérias patógenas nos indivíduos autistas, sendo estes fatores possivelmente ligados às disfunções intestinais apresentadas (Moreno et al., 2015; Jin et al., 2019). Resultados de pesquisas mostram também que o número elevado de Lactobacillus em autistas está ligado a disbiose 
intestinal e a bactéria Desulfovibrio spp. foi associada a severidade de comportamentos restritivos e repetitivos (Tomova et al., 2015; Pulikkan et al., 2018).

Um estudo feito por Rose e colaboradores (2018), afirma que os sintomas gastrointestinais presentes no autismo podem causar desequilíbrio na resposta imune, relacionando também a alterações na parede intestinal. o uso de estratégias nutricionais vem sendo analisadas para a promoção da redução destes sintomas gastrointestinais em indivíduos com autismo, dentre estas se encontram as dietas cetogênicas e isentas de alimentos considerados hiper alergênicos, como glúten e caseína, que provem respostas imunes anormais, havendo o aumento de anticorpos IgG, podendo estar relacionado com o aumento da permeabilidade intestinal, no qual estudos após a intervenção mostraram resultados benéficos em sintomas centrais e periféricos, como comunicação, sociabilidade e linguagem (El-Rashidy et al., 2017; Adams et al., 2018). A suplementação prebióticos e probióticos também vêm sendo uma alternativa para este público podendo impactar no estado comportamental e sintomatologia no TEA (Grimaldi et al., 2017; Sanctuary et al., 2019).

\section{Relação do eixo intestino-cérebro com o autismo}

De acordo com um estudo feito por Eckburg e colaboradores (2005), boa parte dos microrganismos intestinais estão abrigados no cólon, sendo estes em sua maioria anaeróbios estritos, através dos dados fornecidos nesta pesquisa, foi possível afirmar que a microbiota intestinal humana é amplamente diversificada, podendo ter aproximadamente mais de $1 \mathrm{~kg}$ de bactérias, sendo este o peso aproximado de um encéfalo humano.

A composição da microbiota intestinal depende de diversos fatores, como, o tipo de parto, sendo ele normal ou cesariana, alimentação, o uso de antibióticos, genética, estresse ambiental e diminuição de sua diversidade devido às alterações fisiológicas do envelhecimento (Maffei et al. 2017; Wan et al., 2019; Butler et al., 2020). As bactérias presentes no intestino humano pertencem a quatro filos, que são os Firmicutes, Bacteroidetes, Actinobacteria e Proteobacteria, dentre estes filos os mais dominados são os Firmicutes e Bacteriodetes, presentes na microbiota intestinal de um adulto saudável (Cong et al., 2016).

O eixo microbiota intestino-cérebro vem sendo levado em consideração como a causa de transtornos neuropsiquiátricos, visto que estes estão interligados, pois possuem uma comunicação bidirecional entre o sistema nervoso central e o trato gastrintestinal por meio dos mecanismos neuroendócrinos e neuroimunes, um exemplo disto é a capacidade de produção de ácido gama-aminobutírico (GABA) um importante neurotransmissor inibidor presente no sistema nervoso central que pode ser gerado pelas cepas de Lactobacillus rhamnosus YS9, outras cepas também possuem a capacidade de produzir monoaminas, como a dopamina, serotonina e noradrenalina (Shishov et al., 2009; Lin, 2013).

$\mathrm{O}$ equilíbrio do eixo microbiota intestino-cérebro é de suma importância para manter a homeostase, inclusive do Sistema Nervoso Central, havendo sugestões de que este eixo pode implicar no neurodesenvolvimento, no TEA os indivíduos possuem diversas comorbidades gastrointestinais, causando assim um desequilíbrio na microbiota intestinal por conta da redução da integridade da barreira intestinal, ocorrendo uma maior absorção de toxinas e vazamento de ácidos graxos e lipopolissacarídeos, elevando assim o número de biomarcadores inflamatórios, que podem afetar de forma crítica o Sistema Nervoso Central, conforme a Figura 2 (Santocchi et al., 2020; Silva et al., 2020). 
Figura 2: Desequilíbrio no eixo-intestino cérebro.

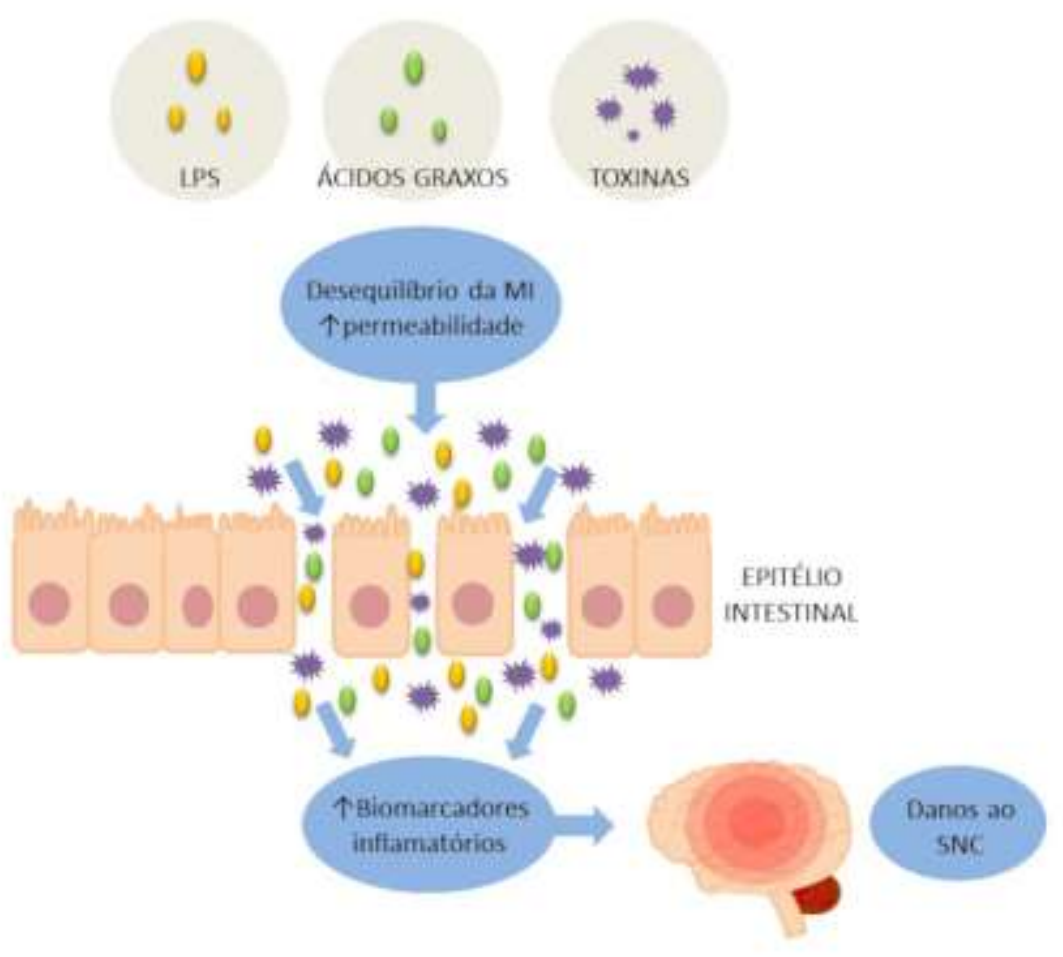

O desequilíbrio na microbiota intestinal aumenta a permeabilidade do epitélio intestinal, ocorrendo maior absorção de toxinas e vazamento de ácidos graxos e lipopolissacarídeos (LPS), que consequentemente elevam o número de biomarcadores inflamatórios, que podem afetar o Sistema Nervoso Central (SNC). Fonte: Autores (2021).

Em um estudo feito por Wang e colaboradores (2019) realizou-se uma análise fecal de 43 crianças autistas e 31 crianças com desenvolvimento típico para identificação de etiópios imunogênicos ligados a microbiota intestinal. Os indivíduos com autismo apresentaram anormalidades na composição da microbiota intestinal sendo associada com níveis elevados de IgA intestinais, mostrando que estes indivíduos possuem possíveis alterações na imunidade intestinal e homeostase, transparecendo que este transtorno pode ter envolvimento com a microbiota por meios das vias imunológicas e mediadores inflamatórios.

Para a melhora de sintomas comportamentais e gastrointestinais no autismo, estudos têm se mostrado bastante pertinentes, realizando terapias de curto e longo prazo com um uso de probióticos, prebióticos e transferências de microbiota para a restauração do equilíbrio da microbiota intestinal, sendo estes possíveis tratamentos para a sintomatologia presente no autismo (Grimaldi et al., 2017; Shaaban et al., 2018; Kang et al., 2019).

A presença de disbiose em autistas também é relatada de forma acentuada. A disbiose intestinal é caracterizada pelo crescimento exacerbado de bactérias no intestino delgado, havendo assim um desequilíbrio na microbiota gastrointestinal, onde se tem o aumento das bactérias patogênicas, os patobiontes, e o menor número de bactérias benéficas, ocorrendo o comprometimento da sua diversidade microbiana (Gagliardi et al., 2018). A mesma também implica na digestão, absorção de nutrientes e metabolismo, podendo impactar no funcionamento cerebral e levar a mudanças comportamentais (Vuong et al., 2017).

\section{Disbiose intestinal no transtorno do espectro autista}

No autismo apesar das alterações gastrointestinais não serem relatadas na sintomatologia são bastante recorrentes neste público, diversos estudos têm relatado esta característica, evidenciando alterações na microbiota de autistas comparada a 
indivíduos controle, notificando que há grandes diferenças na composição microbiana (Strati et al., 2017; Kushak et al., 2017; Zhang et al., 2018; Jin et al., 2019; Ma et al., 2019).

De acordo com um estudo feito por Jin e colaboradores (2019) ao analisar a composição da microbiota fecal, onde utilizou-se o sequenciamento do gene do RNA ribossômico 16S bacteriano (rRNA 16S) de crianças autistas comparadas com crianças neurotípicas, foi possível observar que o grupo com TEA teve menor diversidade da microbiota intestinal do que o grupo controle, a comunidade bacteriana também apresentou alterações, havendo a demasia relativa dos gêneros Lachnoclostridium, Tyzzerella subgrupo 4, Flavonifractor e Lachnospiraceae não identificado e número relativo de Acidaminococcaceae do que os controles saudáveis. Neste outro estudo feito por Zhang e colaboradores (2018), realizaram a comparação da microbiota fecal de indivíduos com TEA e controles, os autistas apresentaram aumento evidentes na razão Bacteroidetes / Firmicutes, havendo de forma relativamente demasiada de Sutterella ,Odoribacter e Butyricimonas, enquanto o aumento de Veillonella e Streptococcus foi menor quando comparado ao grupo controle.

Em este estudo de coorte realizado em infantes autistas e neurotípicos, as alterações na comunidade bacteriana intestinal e fúngica foram evidentes, a maior parte da amostra (90\%), foi classificada com TEA de nível grave, podendo ser notado o impacto da microbiota no Sistema Nervoso Central (SNC), possuindo aumento relevante na proporção Firmicutes / Bacteroidetes em razão do menor número de Bacteroidetes, levando em consideração o gênero, houve a diminuição de Alistipes, Bilophila, Dialister, Parabacteroides e Veillonella, e aumento significativo de Collinsella, Corynebacterium, Dorea e Lactobacillus. Em relação aos níveis de taxa bacteriana se teve um aumento expressivo de Escherichia / Shigella e Clostridium cluster XVIII, a abundância do fungo Candida também foi evidenciada de forma abundante, sendo esta acima do dobro quando comparada com o grupo neurotípico (Strati et al., 2017). Já nos resultados de Ma e colaboradores (2019), houve a análise da microbiota fecal por sequenciamento do gene rRNA 16S de controles e autistas , o grupo com TEA possuiu menor diversidade da microbiota intestinal do que os indivíduos controle e adulteração da microbiota intestinal, com número elevado de Acidaminococcaceae do que nos controles saudáveis, com a diminuição dos gêneros Lachnoclostridium, Tyzzerella subgrupo 4, Flavonifractor e Lachnospiraceae não identificado, sendo observada de forma notória a disbiose microbiana intestina nos indivíduos com TEA.

É possível não só encontrar a presença de disbiose neste público a nível intestinal como também em outros locais, como por exemplo, na cavidade oral, neste estudo feito por Quiao e colaboradores (2018), realizou-se a coleta da microbiota oral tanto de crianças autistas como de controles saudáveis, em seus resultados a diversidade microbiana reduzida foi encontrada nos autistas, contendo a presença de patógenos com número significante, como Haemophilus na saliva $e$ Streptococcus em placas dentárias, e redução de Prevotella, Selenomonas, Acnomyces, Porphyromonas e Fusobacteruim.

\section{Suplementação de prebióticos e probióticos no TEA}

De acordo com a International Scientific Association for Probiotics and Prebiotics, os probióticos são definidos como microrganismos vivos que ao serem administrados em quantidades adequadas, promovem um benefício à saúde do hospedeiro (Marcus et al., 2021). Os mesmos possuem o objetivo de restaurar o equilíbrio da microbiota intestinal, melhorando a barreira intestinal e sua permeabilidade, onde estudos evidenciaram que tanto cepas de Bifidobacterium spp. como Lactobacillus spp. tiveram um papel promissor para a restauração da integridade de células do epitélio intestinal (Guo et al., 2017; Mujagic et al.,2017).

Diversos probióticos podem ter sua função similar com relação à colonização intestinal, reestruturação da microbiota alterada e regulação do trânsito intestinal. Outras espécies utilizadas como probióticos são, a cepa de levedura Saccharomyces boulardii, que secreta proteases e fosfatases, que possuem a capacidade de inativar toxinas patogênicas, elevando os níveis de poliaminas auxiliando na liberação de enzimas fermentativas e maturação dos enterócitos, Clostridium butyricum, sendo este 
um gram-positivo que produz o ácido butírico existente no trato gastrointestinal humano, onde vem sendo utilizado para melhora de doenças inflamatórias ligadas ao intestino e diarreia, e certas espécies de E. coli e Bacillus (World Gastroenterology Organization, 2017; Miyaoka et al., 2017; Sun et al., 2018; García-Collinot et al., 2020).

Estudos evidenciaram que o uso de probióticos pode trazer benefícios em doenças inflamatórias como a síndrome do intestino irritável, neurológicas como Parkinson e Alzeimer, e comorbidades psiquiátricas, como por exemplo, a depressão (Barichella et al., 2016; Tamtaji et al., 2019; Kazemi et al., 2019).

Em relação aos prebióticos, estes são definidos como substratos que são utilizados de forma seletiva por microrganismos hospedeiros, concedendo um benefício à saúde (Marcus et al., 2021). O mesmo irá agir de forma positiva aumentando a colonização de bactérias benéficas presentes, havendo uma modulação da microbiota intestinal (Grimaldi et al., 2017).

Os prebióticos são compostos por polissacarídeos isentos de amido e oligossacarídeos, onde sua maioria é utilizada como ingredientes de alimentos, como por exemplo, em laticínios, cereais, bolachas e chocolate. Os prebióticos mais comuns são, oligofrutose que é presente nos alimentos como por exemplo, trigo; banana, mel e dentre outros, onde esta promove efeitos como a multiplicação de bactérias presentes no cólon, melhora do trânsito intestinal e efeito hipolipemiante, inulina, galactooligossacarídeos, lactulose, que é um dissacarídeo, onde é utilizado para o tratamento de encefalopatia hepática e constipação intestinal, e oligossacarídeos presentes no leite humano (World Gastroenterology Organization, 2017).

Seus benefícios vêm sendo bastante relatados em estudos onde foram utilizados para melhora do estado nutricional de indivíduos com obesidade, melhora de sintomas gastrointestinais em casos de disbiose, diabetes e imunidade (Nicolucci et al., 2017; Ho et al., 2019).

Estudos têm evidenciado os benefícios de probióticos e prebióticos, como neste realizado por Barichella e colaboradores (2016), onde o uso de probióticos e prebióticos tiveram a finalidade de melhorar a constipação presente em pacientes com doença de Parkinson, os resultados mostraram que os indivíduos que ingeriram o leite fermentado com probióticos e prebióticos aumentaram o número de evacuações por semana. Em outro estudo feito por Yazar e Güven (2016), foi avaliado o efeito da suplementação de Zinco e de simbióticos atuando no menor tempo de duração de diarreia infecciosa em crianças, onde ambos ajudaram na redução da mesma.

\section{Conclusão}

É evidente que os sintomas gastrointestinais e comportamentais comprometem a qualidade de vida de pacientes com TEA, nesta revisão integrativa houve evidências de que o uso de prebióticos e probióticos apresentam resultados significativos em crianças com TEA, sendo uma terapia alternativa, promissora e complementar na redução de sintomas gastrointestinais e comportamentais, redução da gravidade do autismo e reversão do quadro de disbiose intestinal. Ainda assim, há a necessidade que mais ensaios clínicos sejam realizados acerca do assunto, pois são escassos e com amostras pequenas.

\section{Referências}

Adams, J. B., Audhya, T., Geis, E., Gehn, E., Fimbres, V., Pollard, E. L., .\& Quig, D. W. (2018). Comprehensive nutritional and dietary intervention for autism spectrum disorder-a randomized, controlled 12-month trial. Nutrients, 10(3), 369. doi: 10.3390/nu10030369.

Almeida, A. K. de A., de Almeida, P. C. F., Oliveira, L. A. , Santos, W. R. C. C., Zagmignan, A., de Oliveira, B. R., ... \& de Carvalho, C. A. (2018). Consumo de ultraprocessados e estado nutricional de crianças com transtorno do espectro do autismo. Revista Brasileira em Promoção da Saúde, 31(3). https://doi.org/10.5020/18061230.2018.7986.

American Psychiatric Association.(2014). DSM-V: Manual diagnóstico e estatístico de transtornos mentais.

Barichella, M., Pacchetti, C., Bolliri, C., Cassani, E., Iorio, L., Pusani, C., ... \& Cereda, E. (2016). Probiotics and prebiotic fiber for constipation associated with Parkinson disease: an RCT. Neurology, 87(12), 1274-1280. doi: 10.1212/WNL.0000000000003127. 
Butler, É. M., Chiavaroli, V., Derraik, J. G., Grigg, CP, Wilson, BC, Walker, N., ... \& Cutfield, WS (2020). Maternal bacteria to correct abnormal gut microbiota in babies born by C-section. Medicine., 99(30). doi: 10.1097 / MD.0000000000021315.

Chistol, L. T., Bandini, L. G., Must, A., Phillips, S., Cermak, S. A., \& Curtin, C.(2018). Sensory sensitivity and food selectivity in children with autism spectrum disorder. Journal of autism and developmental disorders, 48(2): 583-591. doi: 10.1007/s10803-017-3340-9.

Cong, X., Xu, W., Romisher, R., Poveda, S., Forte, S., Starkweather, A., \& Henderson, W. A. (2016). Focus: Microbiome: Gut microbiome and infant health: Brain-gut-microbiota axis and host genetic factors. The Yale journal of biology and medicine, 89(3), 299. Retrieved from https://www.ncbi.nlm.nih.gov/pmc/articles/PMC5045139/.

Eckburg, P. B., Bik, E. M., Bernstein, C. N. , Purdom, E., Dethlefsen, L., Sargent, M., ... \& Relman, D. A. (2005). Diversity of the human intestinal microbial flora. Science, 308(5728), 1635-1638. doi: 10.1126/ science.1110591.

El-Rashidy, O., El-Baz, F., El-Gendy, Y., Khalaf, R., Reda, D., \& Saad, K. (2017). Ketogenic diet versus gluten free casein free diet in autistic children: a case-control study. Metabolic brain disease, 32(6), 1935-1941. https://doi.org/10.1007/s11011-017-0088-z.

Gagliardi, A., Totino, V., Cacciotti, F. , Iebba, V., Neroni, B., Bonfiglio, G., ... \& Schippa, S. (2018). Rebuilding the gut microbiota ecosystem. International journal of environmental research and public health, 15(8), 1679. https://doi.org/10.3390/ijerph15081679.

García-Collinot, G., Madrigal-Santillán, E. O., Martínez-Bencomo, M. A., Carranza-Muleiro, R. A., Jara, L. J., Vera-Lastra, O., ... \& Cruz-Domínguez, M. P. (2020). Effectiveness of Saccharomyces boulardii and metronidazole for small intestinal bacterial overgrowth in systemic sclerosis. Digestive diseases and sciences, 65(4), 1134-1143. https://doi.org/10.1007/s10620-019-05830-0.

Grimaldi, R., Cela, D., Swann, J. R., Vulevic, J., Gibson, G. R., Tzortzis, G., \& Costabile, A. (2017). In vitro fermentation of B-GOS: impact on faecal bacterial populations and metabolic activity in autistic and non-autistic children. FEMS Microbiol Ecol, 93(2):fiw233. https://doi.org/10.1093/femsec/fiw233.

Guo, S., Gillingham, T., Guo, Y., Meng, D., Zhu, W., Walker, W. A., \& Ganguli, K. (2017). Secretions of Bifidobacterium infantis and Lactobacillus acidophilus protect intestinal epithelial barrier function. Journal of pediatric gastroenterology and nutrition, 64(3), 404-412. doi: 10.1097 / MPG.0000000000001310.

Hirota, T., Bishop, S., Adachi M., Shui, A., Takahashi, M., Mori, H., \& Nakamura, K. (2021). Utilization of the Maternal and Child Health Handbook in Early Identification of Autism Spectrum Disorder and Other Neurodevelopmental Disorders. Autism Research, 14, 551-559. https://doi.org/10.1002/aur.2442.

Ho, J., Nicolucci, A. C., Virtanen, H. , Schick, A., Meddings, J., Reimer, R. A., \& Huang, C. (2019). Effect of prebiotic on microbiota, intestinal permeability, and glycemic control in children with Type 1 diabetes. The Journal of Clinical Endocrinology \& Metabolism, 104(10), 4427-4440. https://doi.org/10.1210/jc.2019-00481.

Instituto Brasileiro de Geografia e Estatística. (2010). Censo demográfico 2010.

Jin, J.; Ma, B., Liang, J., et al. (2019). Altered gut microbiota in Chinese children with autism spectrum disorders. Frontiers in cellular and infection microbiology, 9, 40. https://doi.org/10.3389/fcimb.2019.00040.

Kang, D. W., Adams, J. B., Coleman, D. M , Pollard, E. L., Maldonado, J., McDonough-Means, S., ... \& Krajmalnik-Brown, R. (2019). Long-term benefit of microbiota transfer therapy on autism symptoms and gut microbiota. Scientific reports, 9(1), 1-9. https://doi.org/10.1038/s41598-019-42183-0.

Kazemi, A., Noorbala, A. A., Azam, K., Eskandari, M. H., \& Djafarian, K. (2019). Effect of probiotic and prebiotic vs placebo on psychological outcomes in patients with major depressive disorder: a randomized clinical trial. Clinical Nutrition, 38(2), 522-528. https://doi.org/10.1016/j.clnu.2018.04.010.

Kushak, R. I., Winter, H. S., Buie, T. M., Cox, S. B., Phillips, C. D., \& Ward, N. L. (2017). Analysis of the duodenal microbiome in autistic individuals: association with carbohydrate digestion. Journal of pediatric gastroenterology and nutrition, 64(5), 110-116. doi: 10.1097 / MPG.0000000000001458.

Liberati, A., Altman, D. G., Tetzlaff, J., Mulrow, C., Gøtzsche, P. C., Ioannidis, J. P., ... \& Moher, D. (2009). The PRISMA statement for reporting systematic reviews and meta-analyses of studies that evaluate health care interventions: explanation and elaboration. Journal of clinical epidemiology, 62(10), e1-e34. https://doi.org/10.1016/j.jclinepi.2009.06.006.

Lin, Q. (2013).Submerged fermentation of Lactobacillus rhamnosus YS9 for $\gamma$-aminobutyric acid (GABA) production. Brazilian Journal of Microbiology, 44(1), 183-187. https://doi.org/10.1590/S1517-83822013000100028.

Liu, Y. W., Liong, M. T., Chung, Y. C. E., Huang, H. Y., Peng, W. S., Cheng, Y. F., ... \& Tsai, Y. C. (2019). Effects of Lactobacillus plantarum PS128 on children with autism spectrum disorder in Taiwan: a randomized, double-blind, placebo-controlled trial. Nutrients, 11(4), 820. https://doi.org/10.3390/nu11040820.

Ma, B., Liang, J., Dai, M., Wang, J., Luo, J., Zhang, Z., \& Jing, J. (2019). Altered gut microbiota in chinese children with autism spectrum disorders. Frontiers in cellular and infection microbiology, 9, 4. https://doi.org/10.3389/fcimb.2019.00040.

Maffei, V. J., Kim, S., Blanchard IV, E. , Luo, M., Jazwinski, S. M., Taylor, C. M., \& Welsh, D. A. (2017). Biological aging and the human gut microbiota. Journals of Gerontology Series A: Biomedical Sciences and Medical Sciences, 72(11), 1474-1482. https://doi.org/10.1093/gerona/glx042.

Marco, M. L., Sanders, M. E., Gänzle, M Arrieta, M. C., Cotter, P. D., De Vuyst, L., ... \& Hutkins, R..(2021). The International Scientific Association for Probiotics and Prebiotics (ISAPP) consensus statement on fermented foods. Nature Reviews Gastroenterology \& Hepatology, 1-13. https://doi.org/10.1038/s41575-020-00390-5.

Mendes, F. C. V.; Santos, L. C.; Kempinski, E. C. et al., Colla, L., \& Bueno, F. C. (2016) Autismo: caracterização e classificação do grau de severidade dos alunos da associação maringaense dos autistas (ama) com base no método cars. Brazilian Journal of Surgery and Clinical Research - BJSC. 15(3), 31-41. Retrieved from https://www.mastereditora.com.br/periodico/20160804_210918.pdf. 
Miyaoka, T., Kanayama, M., Wake, R., Hashioka, S., Hayashida, M., Nagahama, M., ... \& Horiguchi, J. (2017). Clostridium butyricum MIYAIRI 588 as adjunctive therapy for treatment-resistant major depressive disorder: a prospective open-label trial. Clinical neuropharmacology, 41 (5), 151-155. doi: 10.1097 / WNF.0000000000000299.

Moreno, X. ; Santamaria, G.; Sánchez, R., la Torre, B., Garcés, F., Hernández, C., ... \& López, K. (2015) Microbiota gastrointestinal aeróbica en niños con trastorno del espectro autista. Estudio preliminar. Gen, 69(2). Retrieved from http://ve.scielo.org/scielo.php?script=sci_arttext\&pid=S001635032015000200004

Mujagic, Z., De Vos, P., Boekschoten, M. V. , Govers, C., Pieters, H. J. H., De Wit, N. J., ... \& Troost, F. J. (2017). The effects of Lactobacillus plantarum on small intestinal barrier function and mucosal gene transcription; a randomized double-blind placebo controlled trial. Scientific reports. 7(1), 1-11. https://doi.org/10.1038/srep40128.

Nicolucci, A. C., Hume, M. P., Martínez, I., Mayengbam, S., Walter, J., \& Reimer, R. A. (2017). Prebiotics reduce body fat and alter intestinal microbiota in children who are overweight or with obesity. Gastroenterology, 153(3), 711-722. https://doi.org/10.1053/j.gastro.2017.05.055.

O'connor, K.; Hamm, J. P. \& Kirk, I. J. (2005) The neurophysiological correlates of face processing in adults and children with Asperger's syndrome. Brain and Cognition, 59(1), 82-95. https://doi.org/10.1016/j.bandc.2005.05.004.

Parmeggiani, A., Corinaldesi, A. \& Posar, A. (2019) Early features of autism spectrum disorder: a cross-sectional study. Italian journal of pediatrics, 1, 8-45. https://doi.org/10.1186/s13052-019-0733-8.

Pulikkan, J., Maji, A., Dhakan, D. B., Saxena, R., Mohan, B., Anto, M. M., ... \& Sharma, V. K. (2018). Gut microbial dysbiosis in Indian children with autism spectrum disorders. Microbial ecology, 76(4), 1102-1114. https://doi.org/10.1007/s00248-018-1176-2.

Qiao, Y., Wu, M., Feng, Y. , Zhou, Z., Chen, L., \& Chen, F. (2018). Alterations of oral microbiota distinguish children with autism spectrum disorders from healthy controls. Scientific reports. 8(1), 1-12. https://doi.org/10.1038/s41598-018-19982-y.

Rose, D. R., Yang, H., Serena, G., Sturgeon, C., Ma, B., Careaga, M., ... \& Ashwood, P. (2018). Differential immune responses and microbiota profiles in children with autism spectrum disorders and co-morbid gastrointestinal symptoms. Brain, behavior, and immunity, 70, 354-368. https://doi.org/10.1016/j.bbi.2018.03.025.

Sanctuary, M. R., Kain, J. N., Chen, S. Y., Kalanetra, K., Lemay, D. G., Rose, D. R., ... \& Angkustsiri, K. (2019). Pilot study of probiotic/colostrum supplementation on gut function in children with autism and gastrointestinal symptoms. PloS one, 14(1). https://doi.org/10.1371/journal.pone.0210064.

Santocchi, E., Guiducci, L., Prosperi, M., Calderoni, S., Gaggini, M., Apicella, F., ... \& Muratori, F..(2020). Effects of Probiotic Supplementation on Gastrointestinal, Sensory and Core Symptoms in Autism Spectrum Disorders: A Randomized Controlled Trial. Front Psychiatry, 25;11:550593. doi: 10.3389/ fpsyt.2020.550593

Shaaban, S. Y., El Gendy, Y. G., Mehanna, N. S. , El-Senousy, W. M., El-Feki, H. S., Saad, K., \& El-Asheer, O. M. (2018). The role of probiotics in children with autism spectrum disorder: A prospective, open-label study. Nutritional neuroscience, 21(9), 676-681. https://doi.org/10.1080/1028415X.2017.1347746.

Shishov, V. A., Kirovskaya, T. A., Kudrin, V. S., \& Oleskin, A. V. (2009). Amine neuromediators, their precursors, and oxidation products in the culture of Escherichia coli K-12. Applied biochemistry and microbiology, 45(5), 494-497. https://doi.org/10.1134/S0003683809050068.

Silva, D. V. D., Santos, P. N. M. \& Silva, D. A. V. D. (2020). Excesso de peso e sintomas gastrintestinais em um grupo de crianças autistas. Revista Paulista de Pediatria, 38. https://doi.org/10.1590/1984-0462/2020/38/2019080.

Sociedade Brasileira de Pediatria. (2019) Departamento Científico de pediatria do Desenvolvimento e Comportamento. Transtorno do espectro autista: Manual de Orientação.

Strasser, L.; Downes, M.; Kung, J., Cross, J. H., \& De Haan, M. (2018). Prevalence and risk factors for autism spectrum disorder in epilepsy: a systematic review and meta-analysis. Developmental Medicine \& Child Neurology, 60(1), 19-29. https://doi.org/10.1111/dmcn.13598.

Strati, F., Cavalieri, D., Albanese, D. , De Felice, C., Donati, C., Hayek, J., ... \& De Filippo, C. (2017). New evidences on the altered gut microbiota in autism spectrum disorders. Microbiome, 22;5(1):24. https://doi.org/10.1186/s40168-017-0242-1.

Sun, Y. Y., Li, M., Li, Y. Y. , Li, L. X., Zhai, W. Z., Wang, P., ... \& Li, Y. Q. (2018). The effect of Clostridium butyricum on symptoms and fecal microbiota in diarrhea-dominant irritable bowel syndrome: a randomized, double-blind, placebo-controlled trial. Scientific reports, 8(1), 1-11. https://doi.org/10.1038/s41598-018-21241-z.

Tamtaji, O. R., Heidari-Soureshjani, R., Mirhosseini, N., Kouchaki, E., Bahmani, F., Aghadavod, E., ... \& Asemi, Z. (2019). Probiotic and selenium cosupplementation, and the effects on clinical, metabolic and genetic status in Alzheimer's disease: a randomized, double-blind, controlled trial. Clinical Nutrition, 38(6), 2569-2575. https://doi.org/10.1016/j.clnu.2018.11.034.

Tomova, A., Husarova, V., Lakatosova, S., Bakos, J., Vlkova, B., Babinska, K., \& Ostatnikova, D. (2015). Gastrointestinal microbiota in children with autism in Slovakia. Physiology \& behavior, 138, 179-187. https://doi.org/10.1016/j.physbeh.2014.10.033.

Vuong, H. E., Yano, J. M., Fung, T. C. , \& Hsiao, E. Y. (2017). The microbiome and host behavior. Annual review of neuroscience, 40, 21-49. https://doi.org/10.1146/annurev-neuro-072116-031347.

Wan, Y., Wang, F., Yuan, J. , Li, J., Jiang, D., Zhang, J., ... \& Li, D. (2019). Effects of dietary fat on gut microbiota and faecal metabolites, and their relationship with cardiometabolic risk factors: a 6-month randomised controlled-feeding trial. Gut, 68(8), 1417-1429. http://dx.doi.org/10.1136/gutjnl-2018317609.

Wang, M., Zhou, J., He, F., Cai, C., Wang, H., Wang, Y., ... \& Zhou, W. (2019). Alteration of gut microbiota-associated epitopes in children with autism spectrum disorders. Brain, behavior, and immunity, 75, 192-199. https://doi.org/10.1016/j.bbi.2018.10.006. 
Research, Society and Development, v. 11, n. 1, e12811124061, 2022

(CC BY 4.0) | ISSN 2525-3409 | DOI: http://dx.doi.org/10.33448/rsd-v11i1.24061

Wang, Y., Li, N., Yang, J. J. , Zhao, D. M., Chen, B., Zhang, G. Q., ... \& Gai, Z. T. (2020). Probiotics and fructo-oligosaccharide intervention modulate the microbiota-gut brain axis to improve autism spectrum reducing also the hyper-serotonergic state and the dopamine metabolism disorder. Pharmacological research, 157, 104784. https://doi.org/10.1016/j.phrs.2020.104784.

World Gastroenterology Organization. (2017). Diretrizes Mundiais da Organização Mundial de Gastroenterologia - Probióticos e prebióticos.

World Health Organization. (2020). News room: Autism spectrum disorders.

Yazar, A. S., Güven, Ş. \& Dinleyici, E. Ç. (2016). Effects of zinc or synbiotic on the duration of diarrhea in children with acute infectious diarrhea. Turk J Gastroenterol, 27(1), 537-40. doi: 10.5152/tjg.2016.16396.

Zhang, M., Ma, W., Zhang, J., He, Y., \& Wang, J. (2018). Analysis of gut microbiota profiles and microbe-disease associations in children with autism spectrum disorders in China. Scientific reports, 8(1), 1-9. https://doi.org/10.1038/s41598-018-32219-2. 